\title{
The Value of Cone Beam Computed Tomography in the Management of Dentigerous Cysts - A Review and Case Report
}

\author{
James R Allison BDS(Hons), MFDS RCPS Glas \\ General Professional Trainee, Newcastle Dental Hospital \\ Grace Garlington BDS, MFDS RCPS Glas \\ General Professional Trainee, Newcastle Dental Hospital
}

This is the peer reviewed version of the following article: [Allison, J. and Garlington, G. (2017), The Value of Cone Beam Computed Tomography in the Management of Dentigerous Cysts - A Review and Case Report. Dental Update, 44: 182-188.] which has been published in

final form at [doi:10.12968/denu.2017.44.3.182]. This article may be used for noncommercial purposes in accordance with George Warman Terms and Conditions 


\title{
The Value of Cone Beam Computed Tomography in the Management of Dentigerous Cysts - A Review and Case \\ Report
}

\begin{abstract}
CBCT has recently seen an expansion in use, however there are few robust, evidence-based guidelines to inform practitioners. This article reports the case of a large dentigerous cyst in the maxilla affecting the eruption multiple teeth, considers the use of CBCT in management of such lesions, and discusses guidelines on the use of CBCT in dentistry.
\end{abstract}

Clinical Relevance: As CBCT use increases it is important that practitioners understand the guidelines surrounding its use. Due to the prevalence of dentigerous cysts, it is likely that they will be encountered clinically, and it is important that clinicians referring patients with such lesions are familiar with the principles of managing them.

Objectives: The reader should understand the basic principles of the appropriate use of CBCT in dental and maxillofacial radiology, the presentation of dentigerous cysts and some of the principles of managing them. 


\section{The Value of Cone Beam Computed Tomography in the Management of Dentigerous Cysts - A Review and Case}

\section{Report}

Radiography is important in the diagnosis and management of most oral and dental pathology, and is often invaluable for lesions of the jaw whose precise location and nature may be very difficult to appreciate clinically. For most of the early $20^{\text {th }}$ century, plain film dental radiography was the mainstay, before the introduction in the latter part of the century of tomographic techniques. It wasn't however until around the turn of this century that cone beam computed tomography ( $\mathrm{CBCT}$ ) became commercially available within dentistry, and its use has been increasing over the last decade particularly ${ }^{1}$.

When localising a lesion clinically, cortical expansion may give an indication of whether it lies nearer the buccal or palatal cortical plate and plain film radiography is useful in imaging small lesions, often using parallax techniques. Plain film radiography is cheap and accessible and exposes the patient to a comparably lower dose than other modalities such as CT; however there may be difficulty in fully appreciating spatial relationships where lesions are complex. $\mathrm{CBCT}$ can provide high resolution three dimensional imaging at a much lower dose than conventional multi-detector $\mathrm{CT}$, however the radiation dose received is significantly greater than conventional radiography. The effective dose received from a single intraoral radiograph may be less than 1.5 microSieverts $(\mu \mathrm{Sv})$ when adequate dose reduction techniques are used, compared to between 2.7-24.3 $\mu$ Sv for a dental panoramic radiograph. Doses for CBCT investigations are between 11-674 $\mathrm{SSv}$ for small to medium field dentoalveolar views, and 30-1073 $\mu$ Sv for large field craniofacial views ${ }^{2}$. The reported ranges in dosimetry are largely due to differences in CBCT equipment.

Because of the rapid expansion in the use of $\mathrm{CBCT}$, the evidence base has been slow to keep pace with practice. In 2014 Horner et al. ${ }^{3}$ Undertook a systematic review of guidelines for CBCT use in dental and maxillofacial radiology and found 26 publications which met their inclusion criteria, of which only two were evidence based. The recommendations of the majority of the guidelines identified were that $\mathrm{CBCT}$ should be reserved for use when the 
question for which imaging is required cannot be answered by conventional radiographic techniques alone. The one exception to this was that a small minority of guidelines for CBCT use in implant planning recommended universal use. This was in disagreement with most of the guidelines concerning implant planning where a more selective approach was recommended.

\section{Dentigerous Cysts}

The dentigerous cyst (DC) is the second most common odontogenic cyst of the jaw, after the radicular cyst, and accounts for $20-30 \%$ of all jaw cysts. The cysts are thought to arise from the reduced enamel epithelium of unerupted teeth and are most common in the first to third decades and arising from the third molar, particularly in the mandible ${ }^{4-6}$.

Discovery is often due to chance radiographic finding or investigation into the delayed eruption of a permanent tooth; there may be bony expansion, displacement of affected teeth and pain may be present if the cyst becomes infected. Lesions typically appear radiographically as well corticated, unilocular radiolucencies arising from the cervical region of unerupted teeth ${ }^{7}$. Generally a radiographic follicular enlargement of greater than $3 \mathrm{~mm}$ is suggestive of cystic formation and lesions are usually solitary.

Histologically, lesions typically have a thin, sometimes bilaminar stratified epithelium which may occasionally keratinise by metaplasia. The cyst wall is usually composed of fibrous connective tissue and can be quite vascular, usually with little inflammatory infiltrate. Beyond this there may be a wall of woven bone ${ }^{7}$.

There are several strategies for managing DCs, each with their own merits. Decompression of large lesions may be performed to reduce their size, particularly where there is an intimate relationship to other structures, before further management ${ }^{8,9}$. Marsupialisation may be performed, where the cyst lining is opened and connected to the oral mucosa; this may allow the tooth to be maintained however the entire lining is not available for analysis. Marsupialisation may be appropriate in large cysts where enucleation would affect the integrity of the jaw ${ }^{10}, 11$. Enucleation is the most common approach which involves the extraction of the affected tooth and allows submission of the whole cyst lining for 
histopathological examination. Following treatment, there is usually a low risk of recurrence ${ }^{6}$.

\section{Case Report}

A 9 year boy presented to the Paediatric Dentistry department of Newcastle Dental Hospital after being referred from his general dental practitioner following delayed eruption of the permanent upper left central and lateral incisors. There was a history of dental trauma to the upper left primary central incisor and of removal of a supernumerary tooth in the left anterior maxilla. The medical history was unremarkable. Examination showed a retained and non-mobile ULA and ULB which were discoloured.

Radiographic examination using an upper occlusal view and dental panoramic radiography (DPR) showed a well-defined, corticated, uniformly radiolucent lesion involving the UL1, UL2 and UL3 and enlarged follicles (less than $3 \mathrm{~mm}$ ) associated with the UR3, LL3 and LR3 (Figs. 1.1 1.2). As the extent and origin of this lesion could not be fully visualised using plain film radiography, a CBCT investigation was performed using a Newtom VGi machine (QR SRL, Verona, Italy). The radiographic report showed a well corticated radiolucent lesion surrounding the crown of the UL2 and impeding eruption of the UL1 and UL3 (Figs. 2.1, 2.2, $2.3,2.4)$ most likely a dentigerous cyst arising from one of these teeth. Enlarged follicles associated with the UR3, LL3, LR3, LL7 and LR7 were also noted.

As the lesion involved several teeth and had potential orthodontic implications, an orthodontic opinion was obtained. The consensus was that as the UL2 was of poor prognosis due to its horizontal angulation and proximity to UL3, it would be wise to consider extraction of this tooth. The plan would then involve enucleation of the lesion, submission of the specimen for histopathological analysis, and closed exposure of the UL1 and UL3 using bonded gold chains.

Treatment was carried out under day-stay general anaesthetic. The ULA and ULB were extracted and a mucoperiosteal flap was raised to expose the site. The lesion was found to have already penetrated the buccal cortex of the maxilla and bone was removed to expose the cyst lining (Figs. 3.1-3.3). The lesion was enucleated and was found to be arising from the 
follicle of the UL2 which was extracted (Figs. 3.4-3.5). The UL1 and UL3 were identified, the crowns exposed to their maximum diameter and gold chains were bonded with composite resin (Figs. 3.6-3.8). The flap was replaced and closed with resorbable sutures and the gold chains routed through the sites of the extracted ULA and ULB (Fig. 3.9).

The patient was reviewed from a surgical point of view at 2 weeks, he was without symptoms and healing was progressing well. Histopathological examination of the submitted specimen showed an inflamed cyst, lined by non-keratinised squamous epithelium resembling reduced enamel epithelium fitting with a diagnosis of an inflamed dentigerous cyst.

Orthodontic follow-up was planned with a view to encouraging eruption of the UL1 and UL3 by orthodontic traction and aligning the teeth with fixed appliances. Depending on the relationship of the teeth once they have been brought into the line of the arch, consideration would be made as to whether it is more appropriate to open the space left by the missing UL2 and place a prosthetic replacement or closure of the space and camouflage of the UL3.

\section{Discussion}

The usual presentation of a DC is following the investigation of an unerupted tooth and in this case the eruption of three teeth was affected. The spatial relationship of the teeth was complex and it was not possible to fully appreciate this on plain films. The use of CBCT meant that the relationships of the teeth could be fully appreciated in three dimensions and made the surgery much easier to plan. It also meant that assessing the orthodontic prognosis of the UL2 was easier. This highlights the value of CBCT imaging in cases where plain film radiography is ambiguous.

The risk of using ionising radiation in any patient must be balanced against the clinical benefits, and investigations must only be prescribed where there is likely to be a benefit to the patient's treatment and outcomes. This is especially pertinent when the radiation dose of $\mathrm{CBCT}$ compared to plain film techniques is considered. It is often nice to have three dimensional images to peruse before beginning treatment, but the question remains - does it really benefit the patient, and is that benefit sufficient to offset the risk? It may be easier to arrive at an answer of "yes" in some cases than others; for example for a simple solitary radicular cyst, two periapical views may suffice before root-end surgery, however in the 
present case CBCT allowed more certainty about which tooth was affected and how the lesion related to the teeth. This allowed the surgery to progress more easily, perhaps saving theatre and anaesthetic time, and simplified the consent process as there was more certainty for the patient about which tooth would be lost.

Balancing the risk to benefit ratio is particularly important in paediatric patients, because the lifetime risk of stochastic effects from radiation exposure, such as cancer, is greater with decreasing age. For a certain dose of radiation, the risk to a patient between 10 and 20 may be 2 times greater than for the average 30 year old patient and this is 3 times greater for a patient under $10^{12}$.

The apparent scarcity of robust, evidence-based guidelines despite the widespread use of CBCT is worrying, however the guidelines published by the SEDENTEXCT (Safety and Efficiency of a New and Emerging X-ray Modality) project in $2012^{2}$ are comprehensive, evidence based and scored well compared to other guidelines when assessed using the AGREE II guideline assessment instrument ${ }^{13}$ in the systematic review by Honer et al. ${ }^{3}$. The International Commission on Radiological Protection (ICRP) published guidelines on CBCT more recently ${ }^{14}$, and these were not included in the review by Horner et al.. In agreement with others, the ICRP guidelines recommend that CBCT is used only to answer a specific question compared to other modalities, and that imaging should be optimised by using a small field of view wherever possible.

It is possible that once the cyst had been enucleated, the two remaining teeth would have erupted into place, however exposure may help them erupt faster into the mouth. Since the site was already exposed, if the procedure was found later to have been required, this way a second procedure would be avoided. There appears to be no quality evidence to favour closed or open exposure of palatally displaced canines ${ }^{15}$, and there is a similar paucity of evidence in cases such as the present case. A closed technique was chosen with the intension of maximising the amount of keratinised mucosa surrounding the tooth to optimise the gingival architecture on eruption.

Interestingly in this patient it was noted that the UR3, LL3 and LR3 had enlarged follicles radiographically, although they were not ectopic in position. A supernumerary in the anterior 
maxilla had also been present. This combination of dental abnormalities may suggest a genetic component and it would be wise to review the eruption of these teeth to ensure there is no further follicular enlargement or ectopia. Eruption would be considered late in the maxilla after 12.3 years in girls and 13.1 years in boys ${ }^{16}$, and earlier in the mandible.

Multiple cysts are not common, and have been reported in large case-series at between 1.6\% and $11 \%^{4-6}$ of DCs. Multiple DCs are often thought to be associated with syndromes such as basal cell naevus syndrome, cleidocranial dysplasia, mucopolysaccharidosis, Marteaux-Lamy syndrome and Hunter's syndrome, however in the above studies no multiple DC cases were associated with any syndrome. Case reports of multiple DCs appear to support this finding ${ }^{17-}$ ${ }^{31}$ which is somewhat contrary to popular belief.

\section{Summary}

This case shows the value of CBCT in assessing complex relationships of unerupted teeth, lesions of the jaw and in treatment planning however it highlights the need for adequate justification before such investigations are requested. Evidence-based guidelines do exist for the use of CBCT in dental and maxillofacial radiology such as those produced by SEDENTEXCT and ICRP and these should be used when considering the use of CBCT imaging.

The use of closed versus open exposure is dependent on the operator but either may be beneficial in aligning ectopic or teeth whose eruption has been impeded. The common assumption that multiple DCs are most commonly associated with syndromes may in fact be false.

\section{Declaration}

Appropriate consent was obtained for use of case details, photographic images and radiographs. No funding was received in relation to this article. The authors declare no conflicts of interest.

\section{Acknowledgements}

Thanks to Dr lain Macleod for his assistance, Drs lan Corbett and Rachel Green who performed the surgery and Dr Michelle Moffatt for alerting our attention to the case. 


\section{References}

1. Carter JB, Stone JD, Clark RS, Mercer JE. Applications of Cone-Beam Computed Tomography in Oral and Maxillofacial Surgery: An Overview of Published Indications and Clinical Usage in United States Academic Centers and Oral and Maxillofacial Surgery Practices. J Oral Maxillofac Surg. 2016: 74: 668-79.

2. SEDENTEXCT. Radiation Protection No. 172 Cone beam CT for dental and maxillofacial radiology evidence-based guidelines. Luxembourg: Office for Official Publications of the European Communities; 2012.

3. Horner K, O'Malley L, Taylor K, Glenny AM. Guidelines for clinical use of CBCT: a review. Dentomaxillofac Radiol. 2015: 44: 20140225.

4. Ochsenius G, Escobar E, Godoy L, Penafiel C. Odontogenic cysts: analysis of 2,944 cases in Chile. Med Oral Patol Oral Cir Bucal. 2007: 12: E85-91.

5. Zhang LL, Yang R, Zhang L, Li W, MacDonald-Jankowski D, Poh CF. Dentigerous cyst: a retrospective clinicopathological analysis of 2082 dentigerous cysts in British Columbia, Canada. Int J Oral Maxillofac Surg. 2010: 39: 878-82.

6. Del Corso G, Righi A, Bombardi M, Rossi B, Dallera V, Pelliccioni GA, et al. Jaw cysts diagnosed in an Italian population over a 20-year period. Int J Surg Pathol. 2014: 22: 699-706.

7. Cawson R, Odell E. Cawson's Essentials of Oral Pathology and Oral Medicine, 8th Edition: Churchill Livingstone; 2008.

8. Song IS, Park HS, Seo BM, Lee JH, Kim MJ. Effect of decompression on cystic lesions of the mandible: 3-dimensional volumetric analysis. Br J Oral Maxillofac Surg. 2015: 53: 841-8.

9. Allon DM, Allon I, Anavi Y, Kaplan I, Chaushu G. Decompression as a treatment of odontogenic cystic lesions in children. J Oral Maxillofac Surg. 2015: 73: 649-54.

10. Koca H, Esin A, Aycan K. Outcome of dentigerous cysts treated with marsupialization. J Clin Pediatr Dent. 2009: 34: 165-8.

11. Kirtaniya BC, Sachdev V, Singla A, Sharma AK. Marsupialization: a conservative approach for treating dentigerous cyst in children in the mixed dentition. J Indian Soc Pedod Prev Dent. 2010: 28: 203-8.

12. International Commission on Radiological Protection. Recommendations of the International Commission on Radiological Protection. Annals of the ICRP. 1990: 21: ICRP Publication 60.

13. Brouwers MC, Kho ME, Browman GP, Burgers JS, Cluzeau F, Feder G, et al. AGREE II: advancing guideline development, reporting and evaluation in health care. Cmaj. 2010: 182: E839-42. 
14. International Commission on Radiological Protection. Radiological Protection in Cone Beam Computed Tomography (CBCT). Annnals of ICRP. 2015: 44(1): ICRP Publication 129.

15. Parkin N BP, Thind B, Shah A. Open versus closed surgical exposure of canine teeth that are displaced in the roof of the mouth. Cochrane Database of Systematic Reviews. 2008: Issue 4. Art. No.: CD006966.

16. Husain J, Burden D, McSherry P. Management of the Palatally Ectopic Maxillary Canine. Royal College of Surgeons of England Clinical Guidelines. 2010.

17. Sanjay CJ, David CM, Kaul R, Shilpa PS. Kissing dentigerous cysts involving mandibular canines: report of unusual case with review of literature. J Calif Dent Assoc. 2015: 43: 29-33.

18. Devi P, Thimmarasa VB, Mehrotra V, Agarwal M. Multiple dentigerous cysts: a case report and review. J Maxillofac Oral Surg. 2015: 14: 47-51.

19. Morais HHA, Dias TGdS, Vasconcellos RJdH, Vasconcellos BCdE, Melo AR, Gondim DA, et al. Bilateral mandibular dentigerous cysts: a case report. Revista Gaúcha de Odontologia. 2014: 62: 299-304.

20. Imada TS, Neto VT, Bernini GF, Silva Santos PS, Rubira-Bullen IR, Bravo-Calderon D, et al. Unusual bilateral dentigerous cysts in a nonsyndromic patient assessed by cone beam computed tomography. Contemp Clin Dent. 2014: 5: 240-2.

21. Ishihara $\mathrm{Y}$, Kamioka H, Takano-Yamamoto $\mathrm{T}$, Yamashiro T. Patient with nonsyndromic bilateral and multiple impacted teeth and dentigerous cysts. Am J Orthod Dentofacial Orthop. 2012: 141: 228-41.

22. Tamgadge A, Tamgadge S, Bhatt D, Bhalerao S, Pereira T, Padhye M. Bilateral dentigerous cyst in a non-syndromic patient: Report of an unusual case with review of the literature. J Oral Maxillofac Pathol. 2011: 15: 91-5.

23. Shirazian S, Agha-Hosseini F. Non-syndromic bilateral dentigerous cysts associated with permanent second premolars. Clin Pract. 2011: 1: e64.

24. Saluja JS, Ramakrishnan MJ, Vinit GB, Jaiswara C. Multiple dentigerous cysts in a nonsyndromic minor patient: Report of an unusual case. Natl J Maxillofac Surg. 2010: 1: 16872.

25. Kannan N, Patil R, Sreenivasalu P. Bilateral Maxillary Dentigerous Cysts A Case Report. International Journal of Dental Clinics. 2010: 2.

26. McCrea S. Adjacent dentigerous cysts with the ectopic displacement of a third mandibular molar and supernumerary (forth) molar: a rare occurrence. Oral Surg Oral Med Oral Pathol Oral Radiol Endod. 2009: 107: e15-20.

27. Cury SE, Cury MD, Cury SE, Pontes FS, Pontes HA, Rodini C, et al. Bilateral dentigerous cyst in a nonsyndromic patient: case report and literature review. J Dent Child (Chic). 2009: 76: 92-6. 
28. Fregnani ER, Perez DE, de Carvalho PA, Alves FA. Metachronous bilateral dentigerous cysts associated with permanent first molars. J Dent Child (Chic). 2008: 75: 197-200.

29. Shahrabi Farahani S, Lotfalian M. A pigmented dentigerous cyst in a patient with multiple dentigerous cysts of the jaws: a case report. J Contemp Dent Pract. 2007: 8: 85-91.

30. Tournas AS, Tewfik MA, Chauvin PJ, Manoukian JJ. Multiple unilateral maxillary dentigerous cysts in a non-syndromic patient: A case report and review of the literature. International Journal of Pediatric Otorhinolaryngology Extra. 2006: 1: 100-6.

31. Freitas DQ, Tempest LM, Sicoli E, Lopes-Neto FC. Bilateral dentigerous cysts: review of the literature and report of an unusual case. Dentomaxillofac Radiol. 2006: 35: 464-8. 


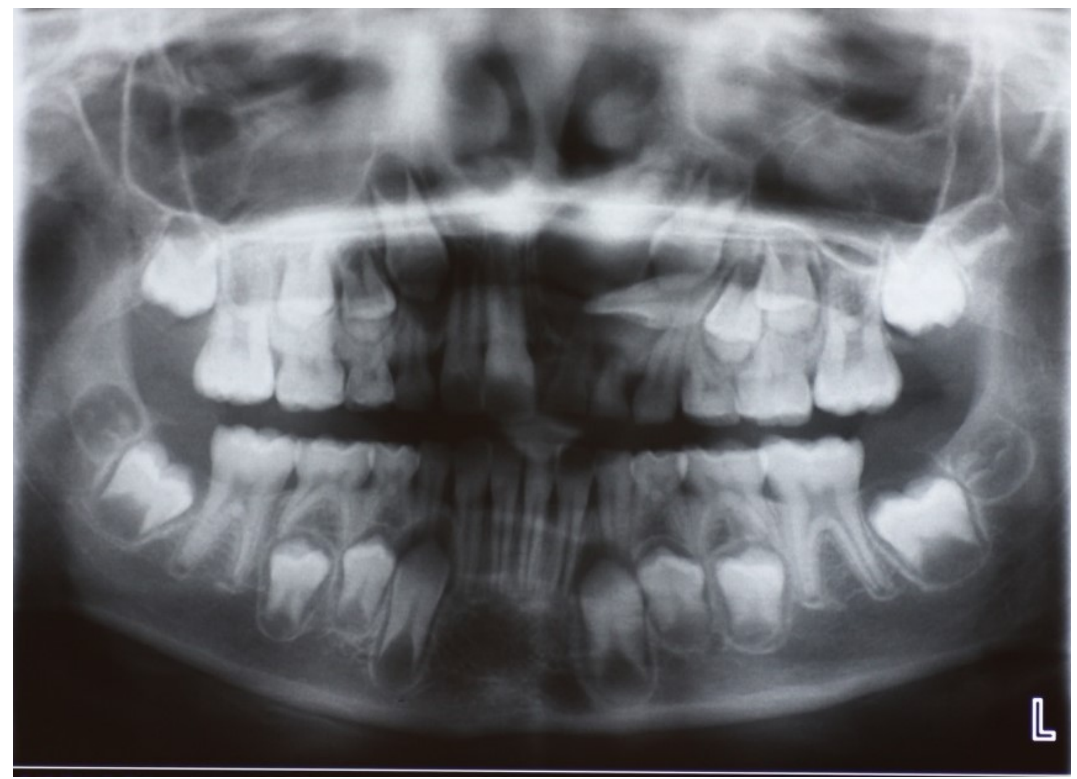

Figure 1.1 - Dental panoramic radiograph showing cystic lesion in the left anterior maxilla.

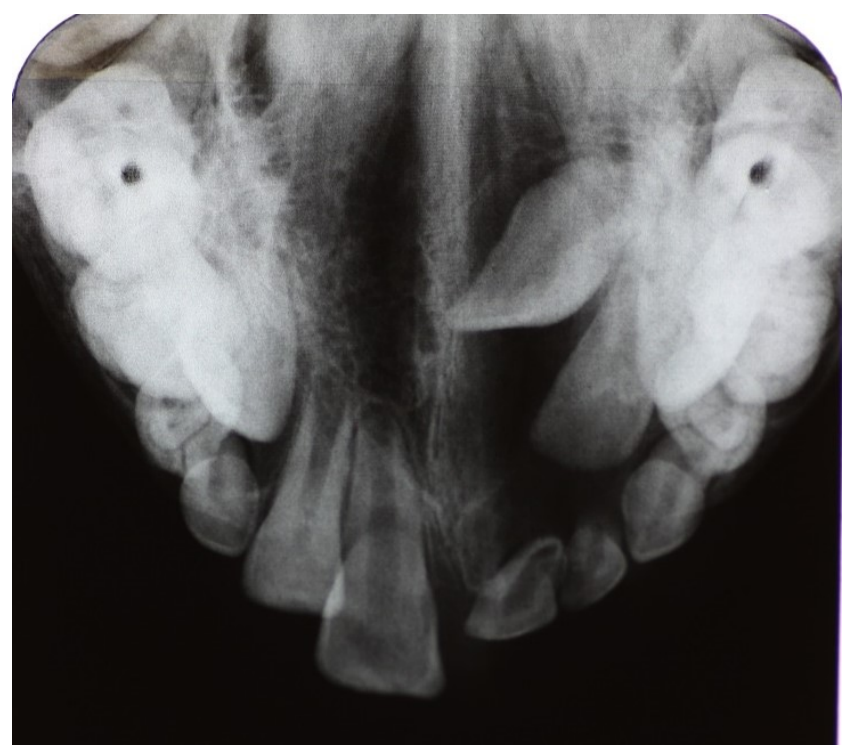

Figure 1.2 - Upper occlusal radiograph showing cystic lesion in the left anterior maxilla.

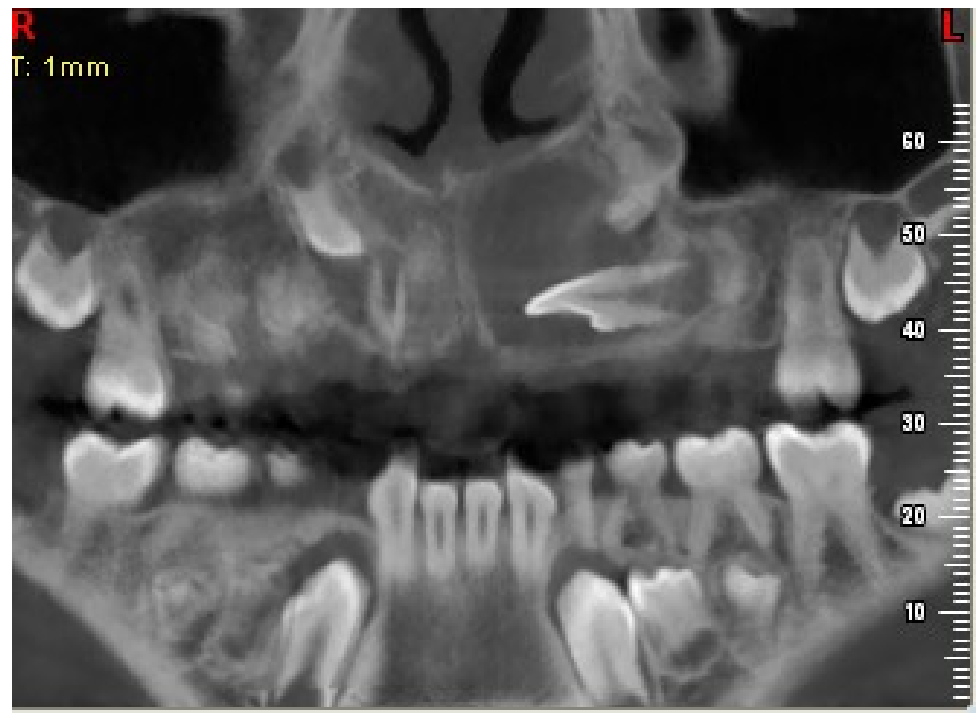

Figure 2.1 - Multiplanar reconstruction of CBCT image of a cystic lesion in the left maxilla. The horizontally placed UL2 is visible.

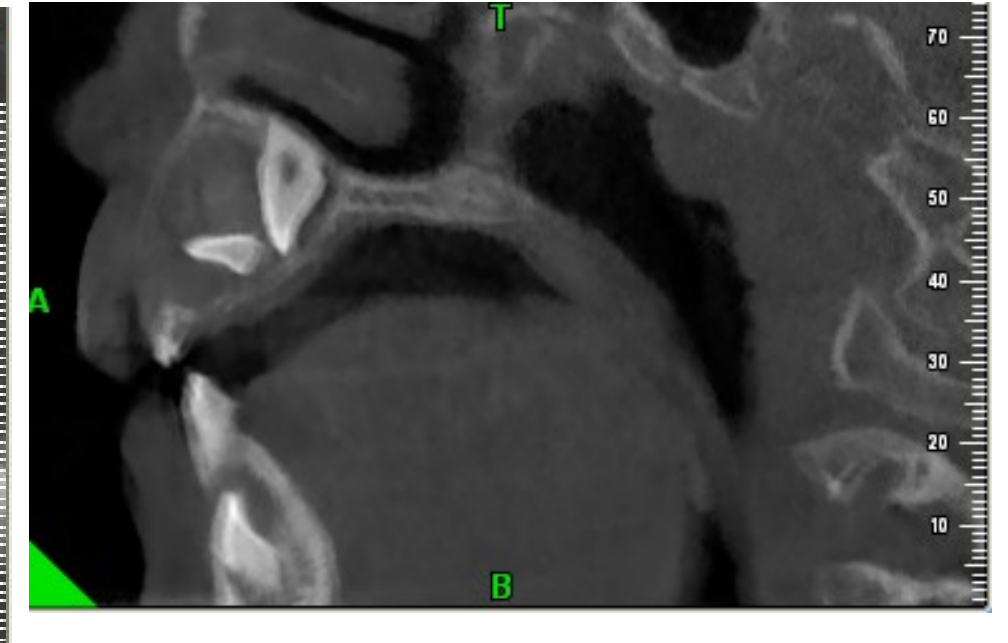

Figure 2.2 - Sagittal section of CBCT image of left maxillary cyst. The crowns of UL1 and the horizontal UL2 are visible. 


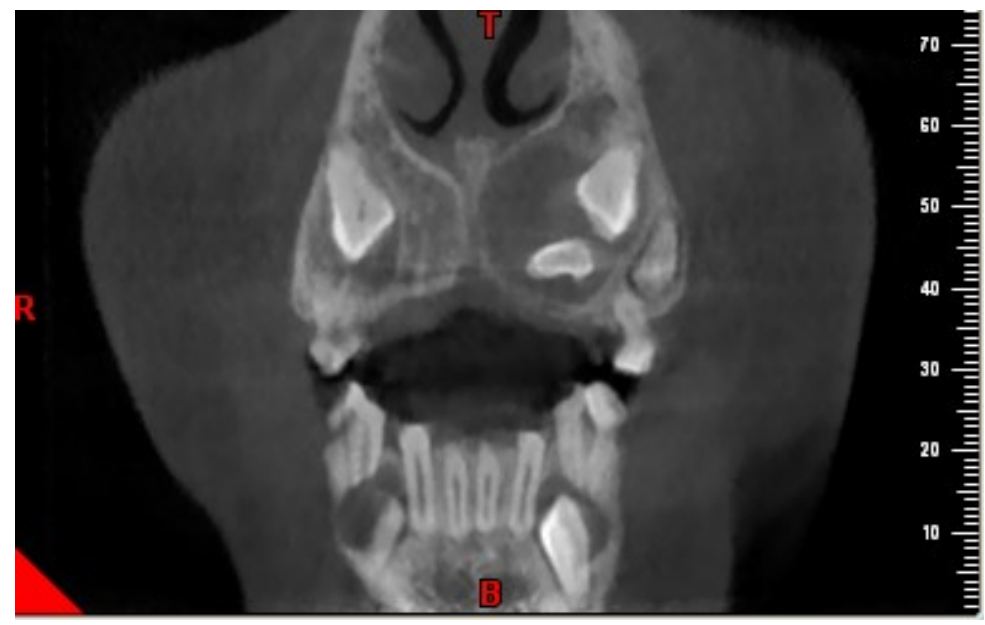

Figure 2.3 - Coronal section of CBCT image of left maxillary cyst. The crowns of the horizontal UL2 and the UL3 visible.
Figure 2.4-Multiplanar reconstruction of CBCT image of a cystic lesion in the left maxilla. The UL2 and are UL3 visible.

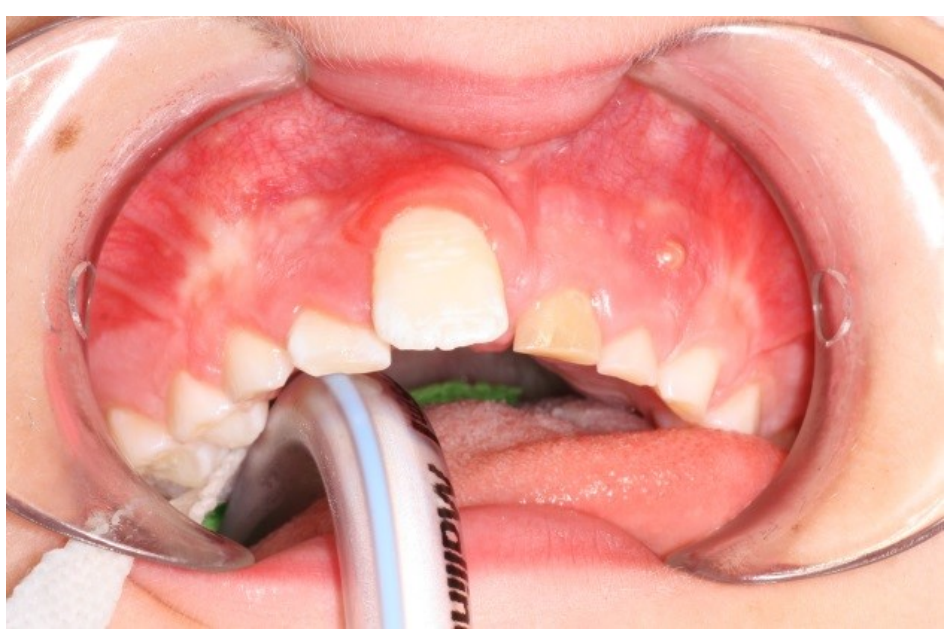

Figure 3.1 - Preoperative Appearance

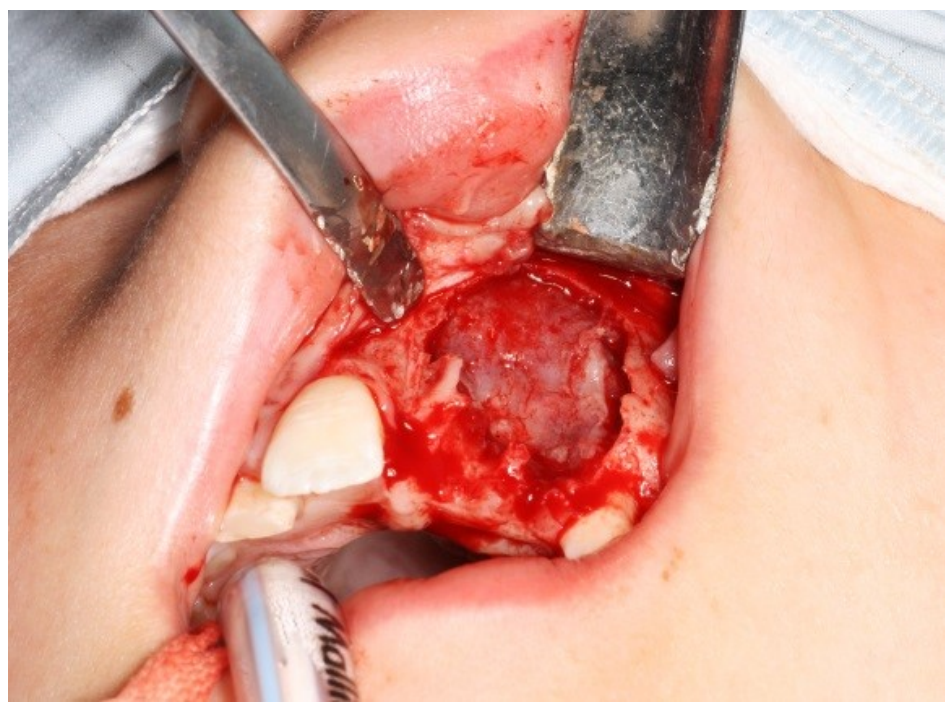

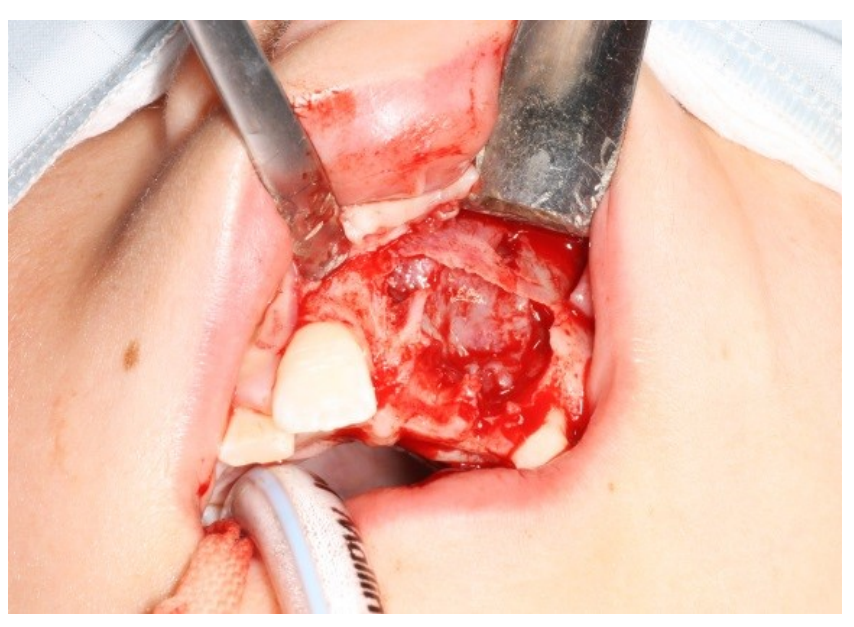

Figure 3.2-Mucoperiosteal flap elevated

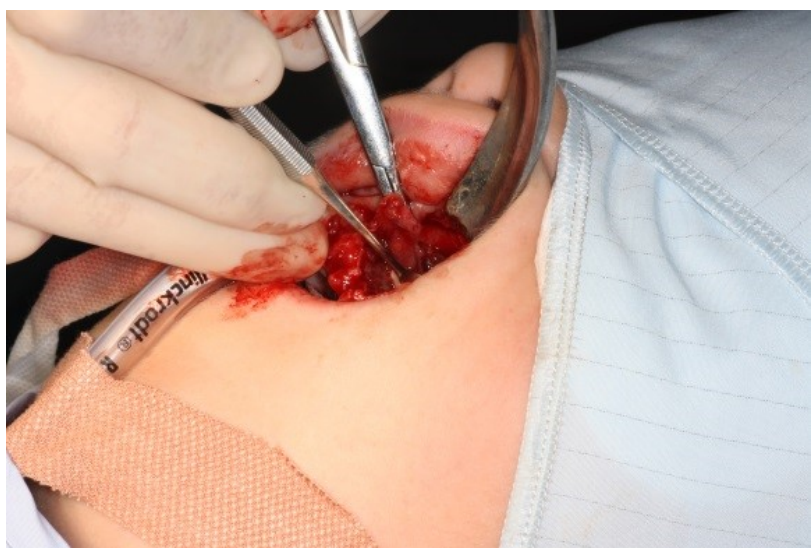

Figure 3.4-Mobilisation of cyst lining

Figure 3.3 - Overlying bone removed 


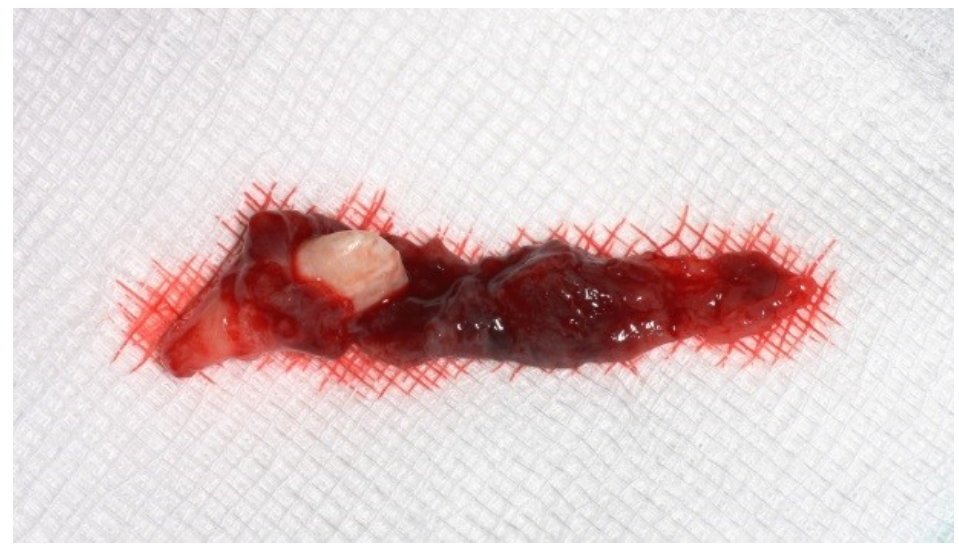

Figure 3.5 - Extracted UL2 and cyst lining which became perforated during enucleation.

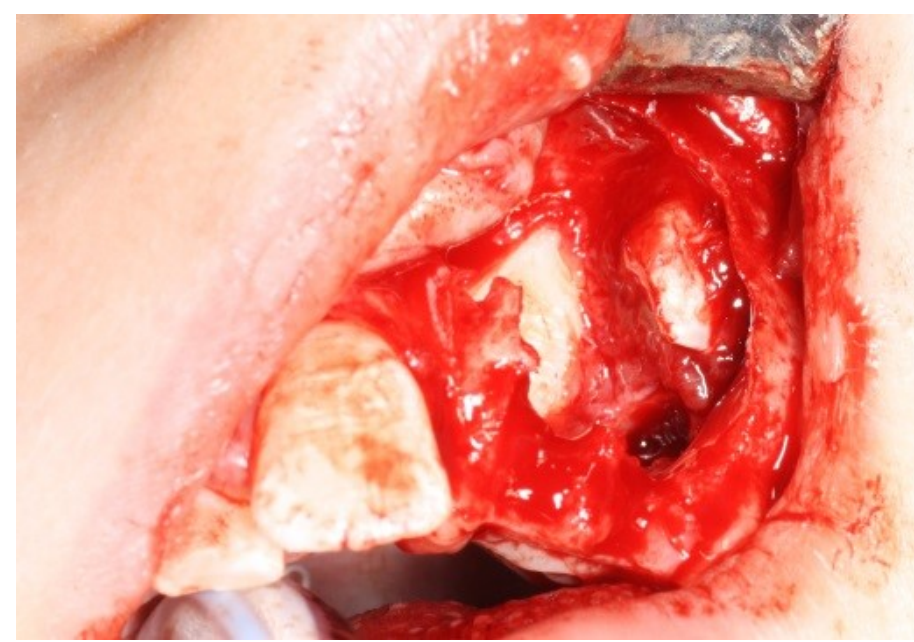

Figure 3.7 - Appearance of UL3 (follicle removed)

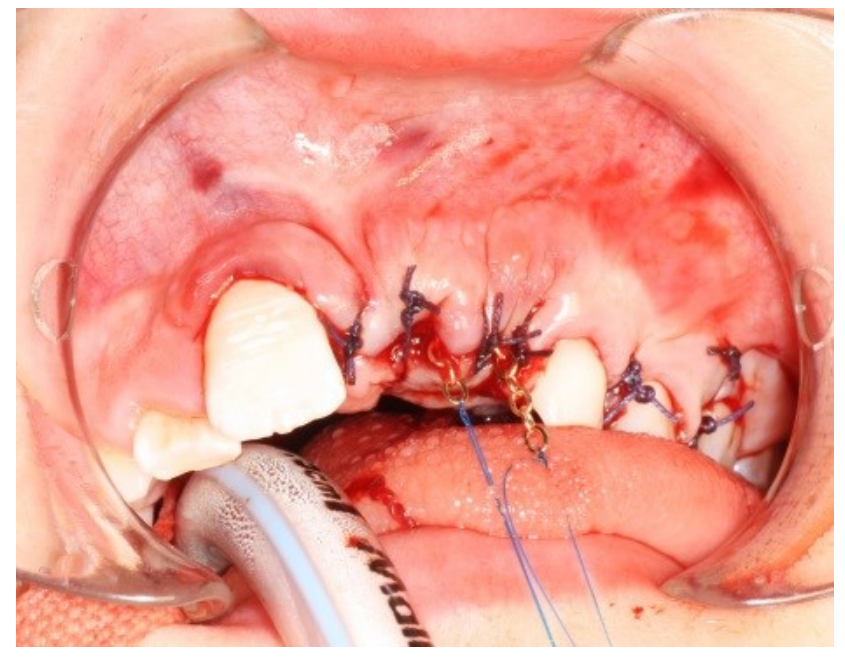

Figure 3.9-Postoperative appearance

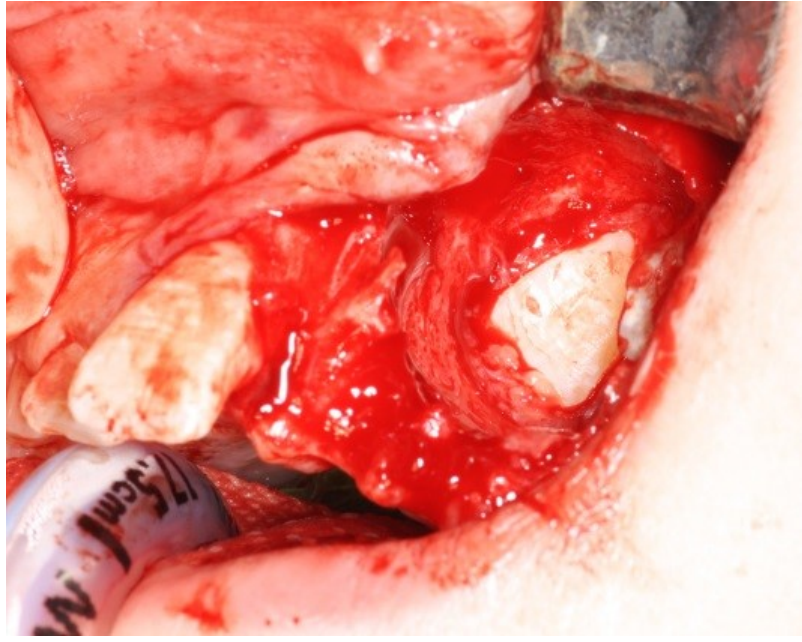

Figure 3.6-Appearance of UL1 (follicle removed)

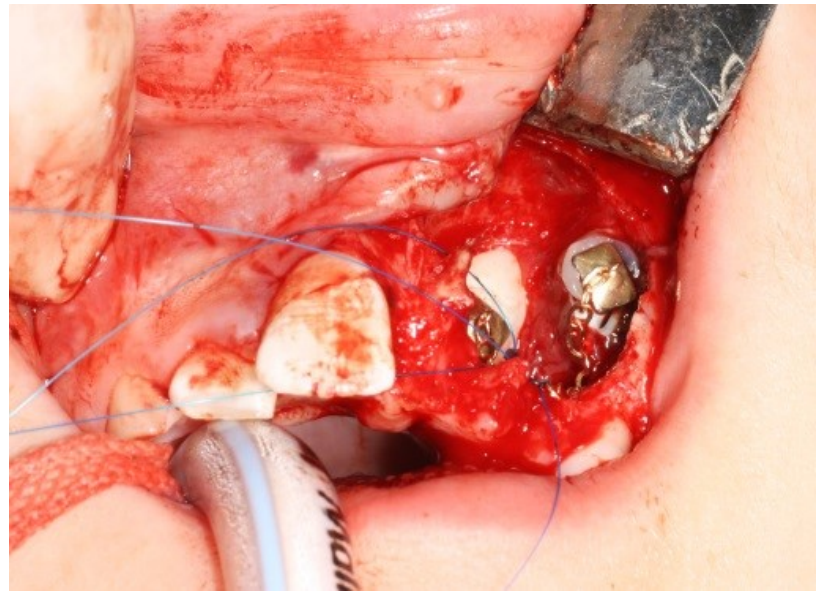

Figure 3.8 - Gold chains bonded to UL1 and UL3 